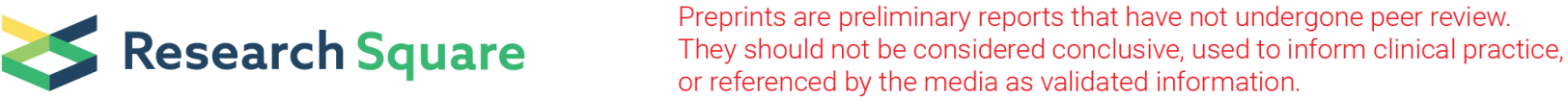

\section{Deep Learning Algorithmic Approach for Operational Anomaly Based Intrusion Detection System in Wireless Sensor Networks}

Ashwini Bhaskar Abhale ( $\sim$ ashwiniabhale@gmail.com )

VIT University https://orcid.org/0000-0002-6053-2282

S S Manivannan

Vellore Institute of Technology: VIT University

\section{Research Article}

Keywords: Deep Convolution Neural Network, Deep Recurrent Neural Network, Deep Short Long Term Memory, Deep Gated Recurrent Unit, Artificial Neural Network, Anomaly Based IDS, Signature Based IDS. Wireless Sensor Network (WSN).

Posted Date: September 21st, 2021

DOI: https://doi.org/10.21203/rs.3.rs-777010/v1

License: (c) (i) This work is licensed under a Creative Commons Attribution 4.0 International License. Read Full License 


\section{Abstract}

Because of the ever increasing number of Internet users, Internet security is becoming more essential. To identify and detect attackers, many researchers utilized data mining methods. Existing data mining techniques are unable to provide a sufficient degree of detection precision. An intrusion detection system for wireless networks is being developed to ensure data transmission security. The Network Intrusion Detection Algorithm (NIDS) uses a deep classification system to classify network connections as good or harmful. Deep Convolution Neural Network (DCNN), Deep Recurrent Neural Network (DRNN), Deep Long Short-Term Memory (DLSTM), Deep Convolution Neural Network Long Short-Term Memory (DCNN LSTM), and Deep Gated Recurrent Unit (DGRU) methods that use NSLKDD data records to train models are proposed. The experiments were carried out for a total of 1000 epochs. During the experiment, we achieved a model accuracy of more than 98 percent. We also discovered that as the number of layers in a model grows, so does the accuracy.

\section{Introduction}

Wireless Sensor Networks (WSNs) are regarded low-power processing candidates with deplorable characteristics. They are employed in a variety of fields to collect data on human activities and behaviour, to monitor diverse natural activities, and so on. WSNs' primary concern is security. There are two major categories: active and passive. Passive attacks, on the other hand, are undetected and use the link above to store data; alternatively they disable the internet's performance factor. Passive attack types include broken nodes, tampering, and traffic. The network's essential attacks on itself during an active attack, as well as the rationale for attacks, may be thus and can also be recognised. Services may be disrupted or corrupted for a period of time as a result of these attacks. Numerous attack types are classified as jamming, hole-attacks, Denial-of-Service (DoS) attacks, Sybil attacks, and flooding. The network's activity is either passive or active. Intrusion Detection Systems (IDSs) provide several pieces of information to other supporting systems, including the detection and location of the intruder, the intrusion instance, the type of intrusion, and the location of the intrusion. As additional information about an intrusion is revealed, this knowledge can be beneficial in reducing and resolving the cause of the attack. Thus, intrusion detection is beneficial for network security. Wireless Networks are regarded as a complex and time-consuming process in terms of performance and optimization. WSN must do the entire set of operations. WSN requires network resources as well as power for a time period during which the WSN must communicate with a centralised or remote base station to perceive the data and do subsequent analysis. With the addition of intrusion detection, WSN focuses on Quality of Service (QoS) and Network Security in order to prevent DoS attacks (Denial of Service). The WSN considers packet forwarding, data collecting, and target tracking to be integral objectives [1].

The building of a reliable and efficient Network Intrusion Detection System is one of the most serious issues in network security. Despite considerable advances in NIDS technology, most solutions still use less capable signature-based methods to identify threats rather than anomaly detection techniques. The system's resistance to change is due to a high false error rate (and related costs), the difficulty in 
collecting accurate training data, training stability, and the system's behavioural dynamics. The present situation will improve to the point when such processes will become inefficient and result in erroneous detection[2]. The aim of this challenge is to develop a generally recognised method for identifying anomalies that can overcome the constraints imposed by current network development. The issue of network security is exacerbated by three main limitations. The first is the network data volume's continuing fast increase. Digital transformation, the fast development of the Internet of Things, and the growing trend of cloud-based services are all driving this growth. Data analytics that are quicker, more cost-effective, and more efficient are needed to cope with huge quantities of data. The second reason is that in order to maximise efficacy and accuracy, granularity and in-depth monitoring are needed. A change away from abstract and high-level observations is required for the NIDS analysis to become more thorough and relevant. Particular network components, such as specific users, operating system versions, or protocols, must, for example, be readily linked to behavioural changes. The variety of data sent by contemporary networks, as well as the development of various protocols, is the fourth factor. The most difficult and complex aspect of the procedure is determining the difference between normal and unethical behaviour. It makes defining a precise standard more difficult and increases the danger of exploitation or zero-day attacks. Machine learning classification methods have been extensively utilised in NIDS research in recent years[3]. Increased detection accuracy has often resulted from the use of these methods. On the other hand, these methods have drawbacks, such as the high degree of human expert interaction needed to analyse data, such as identifying significant data and patterns. Similarly, operating in a diverse and dynamic environment may require a large amount of data (as well as time overheads), which may be difficult. Deep learning is a field of study that will interest attention across a broad range of domains in order to address the issues mentioned above. This is a considerably more sophisticated type of neural network that solves some of shallow learning's flaws. So far, fundamental research has demonstrated that deep learning's superior layer-wise feature learning methods outperform or equal shallow learning techniques. It can gain insight into network data and detect abnormalities more rapidly [4]. We provide a viable deep learning model for NIDS deployment on existing networks in this article. We provided various multilayer deep learning methods to improve the model's accuracy, and we tested it for 1000 epochs and found that it can correctly analyse a broad variety of network data. We used GPUaccelerated TensorFlow[5] to verify our model and obtained encouraging results while analysing the NSLKDD datasets [6].

Table 1 summarises the abbreviations used in this article. 
Table 1

Summarization of abbreviations used in this article

\begin{tabular}{|c|c|c|c|}
\hline Abbreviations & Description & Abbreviations & Description \\
\hline $\mathrm{AE}$ & Acoustic Emission & GPU & $\begin{array}{l}\text { graphics processing } \\
\text { unit }\end{array}$ \\
\hline Al & Artificial Intelligence & GRU & Gated Recurrent Unit \\
\hline AIDS & $\begin{array}{l}\text { Anomaly Base Intrusion Detection } \\
\text { System }\end{array}$ & GRUIDS & $\begin{array}{l}\text { Gated Recurrent Unit } \\
\text { Intrusion detection } \\
\text { system }\end{array}$ \\
\hline ANN & Artificial Neural Network & HIDS & $\begin{array}{l}\text { Host-based Intrusion } \\
\text { detection system }\end{array}$ \\
\hline AUC & Area Under The ROC Curve & ICT & $\begin{array}{l}\text { Information and } \\
\text { communications } \\
\text { technology }\end{array}$ \\
\hline BGP & Border Gateway Protocol & IDS & $\begin{array}{l}\text { Intrusion detection } \\
\text { system }\end{array}$ \\
\hline $\mathrm{CIC}$ & Canadian Institute For Cyber security & IG & information gain \\
\hline CNN & Convolutional Neural Network & LSTM & Long short memory unit \\
\hline CPU & Central Processing Unit & ML & Machine learning \\
\hline CSV & Comma-Separated Values & MLP & Multilayer Perceptron \\
\hline DCGAN & $\begin{array}{l}\text { Deep Convolutional Generative } \\
\text { Adversarial Networks }\end{array}$ & NIDS & $\begin{array}{l}\text { Network-based } \\
\text { Intrusion detection } \\
\text { system }\end{array}$ \\
\hline DCNN & Deep Convolutional Neural Networks & PSO & $\begin{array}{l}\text { Particle Swarm } \\
\text { Optimizations }\end{array}$ \\
\hline DCNNIDS & $\begin{array}{l}\text { Deep Convolutional Neural Network } \\
\text { Intrusion Detection System }\end{array}$ & R2L & Remote to User attack \\
\hline DGRU & Deep Gated Recurrent Unit & RAM, & $\begin{array}{l}\text { Random access } \\
\text { memory }\end{array}$ \\
\hline DL & Deep Learning & RNN & $\begin{array}{l}\text { Recurrent Neural } \\
\text { network }\end{array}$ \\
\hline DLSTM & A Deep Long Short-Term Memory & ROC & $\begin{array}{l}\text { Receiver Operating } \\
\text { Characteristic Curve }\end{array}$ \\
\hline DNN & Deep Neural Networks & SIDS & $\begin{array}{l}\text { Signature-Based } \\
\text { Intrusion Detection }\end{array}$ \\
\hline DOS & Denial-Of-Service & SMOTE & $\begin{array}{l}\text { Synthetic Minority Over- } \\
\text { Sampling Technique }\end{array}$ \\
\hline
\end{tabular}




\begin{tabular}{|llll|}
\hline Abbreviations & Description & Abbreviations & Description \\
\hline ELM & Extreme Learning Machine & SRU & $\begin{array}{l}\text { Simple Recurrent Unit } \\
\text { Based }\end{array}$ \\
\hline FAR & False Acceptance Rate & SVM & $\begin{array}{l}\text { Support Vector } \\
\text { Machine }\end{array}$ \\
\hline FLN & Fast Learning Network & TB & Terabytes \\
\hline FN & False Negative & TN & True Negative \\
\hline FP & False Positive & TPR & True Positive Rate \\
\hline FPR & False Positive Rate & U2R & User to Root Attack \\
\hline FSL & Few-Shot Learning & WSN & $\begin{array}{l}\text { Wireless Sensor } \\
\text { network }\end{array}$ \\
\hline FRR & False Rejection Rate & XGB & $\begin{array}{l}\text { Extreme Gradient } \\
\text { boosting }\end{array}$ \\
\hline
\end{tabular}

The sections of this paper is structured as follows. Section II presents relevant basic background information on IDS system. Section III examines various Al methods IDS in NIDS and existing work on techniques. Section IV specifies evaluation matric on basis of dataset in Section V. represent the result and proposed models. Finally the paper concludes in Section VI.

\section{li. Basic Concepts}

\subsection{Intrusion Detection System}

The term "intrusion detection system" is abbreviated as "IDS." This phrase combines the terms "intrusion" and "detection system." Intrusion is defined as unauthorised access to data held on a computer or network system with the aim of jeopardising its integrity, confidentiality, or availability. A "detection system" is a kind of security method for detecting unlawful actions such as these. An intrusion detection system (IDS) is a kind of security instrument that analyses host and network traffic for any unexpected behaviour that may jeopardise the security policy's confidentiality, integrity, or availability. If malicious behaviour is discovered, the IDS will alert the host or network administrators [1]. As illustrated in Fig. 1, an NIDS is installed on a network and linked to a network switch using port mirroring technology. The aim is to use a network intrusion detection system (NIDS) to mirror all network traffic, both incoming and outgoing, for traffic monitoring and intrusion detection. In addition, NIDS may be installed between the firewall and the network switch, enabling all traffic to flow through.

\subsection{Classification of IDS}

IDS are classified based on how they are deployed or detected. A classification taxonomy is depicted in Fig. 2. 
IDS is further categorised as host-based-IDS (HIDS) or network-based-IDS (NIDS) from the standpoint of deployment. HIDS is deployed on a single data host. Its goal is to monitor everything that occurs on this single host and search for security policy breaches as well as suspicious behaviour. The major drawback is that it must be deployed on all hosts that need intrusion protection, increasing the processing overhead on each node and lowering the IDS's performance. NIDS, on the other hand, is a network security system that protects all devices as well as the whole network from attacks. The NIDS will monitor network traffic in real time for any security breaches and violations. This article [8] discusses the different NIDS techniques. In terms of detection, IDS are categorised as "SIDS(Signature-based intrusion detection)." [9], as well as "AIDS (Anomaly Detection-Based Intrusion Detection)". [10]. SIDS is also known as "misuse intrusion detection" or "knowledge-based intrusion detection." It is built on the idea of creating a signature for an assault pattern. These signatures are kept in a signature database and matched to data patterns to identify attacks. Because signatures for known attacks are accessible, the benefit includes a high detection efficiency for such assaults. However, since it lacks signature patterns, this approach is incapable of detecting new assaults. This technique also requires the upkeep of a huge signature database and the comparison of data packets for potential intrusions, making it a time-consuming process. AIDS, also known as "behavior-based IDS," aims to provide a clear picture of typical activities. Anomaly or aberrant behaviour refers to any deviation from this typical profile. Two of AIDS' most important benefits are its capacity to identify unknown and novel assaults, as well as the changeable form of the typical activity profile for various networks and applications. The high FAR is the biggest drawback, as it makes it impossible to differentiate between typical and abnormal intrusion detection patterns. The adoption of the loT paradigm has led in an exponential rise in the usage of loT devices as a consequence of improvements in network technology. The WSN, which comprises of a collection of datagathering sensor nodes, is a critical component in the loT network's growth. These Internet of Things sensor devices gather a huge quantity of vital data, which is subsequently transmitted over the internet. This enormous data, coupled with the WSN's complicated structure of resource-constrained sensor nodes, presents a security concern to the loT network. Intrusion detection systems (IDS) are generally considered to be one of the most effective methods for loT and WSN security in this respect. A number of IDS methods have been suggested in the literature, all of which depend on successful use of watchdogs, trust models, and game-theoretic ideas. The data traffic of neighbouring nodes is monitored and logged by network nodes known as watchdogs. After that, a judgement is taken regarding the misbehaving nodes based on a set of rules. A variety of methods for anomaly and intrusion detection utilising watchdogs have been suggested in the WSN sector. Another way to improve an IDS's effectiveness is to utilise trust models. An IDS based on the trust model evaluates their nodes' trustworthiness by constantly monitoring network traffic for anomalous behaviour in order to detect malicious nodes. We'll look at Al-based NIDS in this post, which may be used to protect a WSN network by limiting access going via the edge router.

\section{lii. Ai Methods For Nids}

This section covers both the overall process of developing Al-based NIDS as well as the details of the most often utilised deep learning methods for developing successful NIDS. Deep learning employs both 
supervised and unsupervised methods. Supervised algorithms extract useful information from tagged data. Unsupervised algorithms, on the other hand, extract relevant information and features from unlabeled data. DL is an artificial intelligence discipline that includes all methods and algorithms that enable computers to learn autonomously by extracting relevant facts from huge datasets using mathematical formulae.

\subsection{Artificial Neural Network}

The human nervous system provides the basis for ANN, which is a supervised algorithm. It is made up of processing components called neurons (nodes) and the connections that link them. These nodes are separated by an input layer, multiple hidden layers, and an output layer. For the ANN, the back propagation method is employed as a learning approach. The capacity of an ANN method to conduct nonlinear modelling by learning from bigger datasets is its primary benefit. The major issue with training the ANN model is that it takes a long time due to its complexity, slowing down the learning process and resulting in a poor result. To address the limitations of ANNs, Huang et al [11] proposed a novel ANN termed a gradient boosting machine (ELM). The ELM is a single hidden layer feed-forward neural network that uses the input weights and hidden layer bias at random to calculate output weights analytically. Based on the ELM idea, Li et al [12] created a Fast Learning Network (FLN). FLN is made up of a parallel connection of a multilayer feed-forward neural network and a single-layer feed-forward neural network. FLN showed good performance and stability while utilising fewer hidden nodes and requiring less time. Ali et al [13] developed and evaluated a model based on FLN and particle swarm optimization (PSO-FLN) to solve the IDS issue using the KDD Cup'99 dataset. Against verify the model, the FLN was compared to a variety of optimization methods. PSO-FLN beats other FLN models using a variety of optimization techniques. They also found that increasing the number of neurons in the buried layer increases accuracy. The major disadvantage is that for lower assault classes, the detection rate accuracy is lower.

\subsection{Deep Learning Algorithms}

$\mathrm{DL}$ is a subset of machine learning that uses many hidden layers to attain the characteristics of a deep network. These methods are more efficient than machine learning because of their deep structure and capacity to learn important characteristics from a dataset on their own and give an output. This section discusses the DL techniques that were utilised to build NIDS solutions.

\subsubsection{Recurrent neural networks}

Recurrent Neural Networks (RNN) are used to represent sequence data and enhance the capabilities of feed-forward neural networks. The RNN is made up of three kinds of units: input, hidden, and output, with the hidden units acting as memory. Each RNN unit makes a judgement depending on the output of the previous input and the current input. RNNs are utilised in a variety of applications, including speech processing, human activity identification, handwriting prediction, and semantic comprehension. RNNs may be utilised in an IDS for supervised classification and feature extraction. While RNNs are capable of managing short sequences, when the sequence is too lengthy, difficult with short-term memory. Several 
RNN variations, such as the long short-term memory (LSTM) and gated recurrent unit, have been suggested to solve these issues (GRU).

Using RNN-based IDS, Yin et al. [14] suggested categorising the NSL-KDD dataset into binary and multiclass classes. A variety of hidden nodes and learning rates were used to test the model. The results indicate that the model's accuracy is influenced by learning rates and the amount of hidden nodes. The greatest accuracy was obtained with 80 hidden nodes and learning rates of 0.1 and 0.5 for binary and multiclass instances, respectively. A reduced-size RNN model was suggested by Sheikhan et al. [15]. The primary drawback of this study is the extra computer processing needed, which results in a longer model training time and a lower detection rate for the R2L and U2R classes. In addition, the paper lacks a comparison of the proposed model's performance to that of other deep learning methods. To develop an RNN-based IDS with GRU as the main memory, Xu et al. integrated an RNN-based IDS with GRU as the primary memory, as well as a multilayer perceptron and a softmax classifier. The suggested approach was tested using the KDD Cup' 99 and NSL-KDD datasets. When compared to other methods, the detection rates were quite high, according to the findings of the experiments. Their model's major weakness is that it identifies fewer assaults from uncommon attack classes such as U2R and R2L. Naseer et al. [16] compared IDS based on a range of deep learning and machine learning methods and implemented on a graphics processing unit-based testbed. The NSL-KDD dataset is used as a benchmark, and the findings indicate that LSTM and Deep CNN models are more accurate than other models.

\subsubsection{Convolutional neural network}

CNNs are a kind of deep learning (DL) structure that works well with array-based data. It comprises three layers: an input layer, a feature extraction layer made up of a stack of convolutional and pooling layers, and a classification layer made up of a fully connected layer and a softmax classifier. CNN is well-known and recognised in the area of computer vision. They are utilised in the IDS to conduct supervised feature extraction and classification.

A CNN-based efficient IDS was proposed by Xiao et al. [17]. To extract features, the fundamental concept is to start with PCA and AE. After converting the one-dimensional vector (feature set) to a twodimensional matrix, the convolutional Neural Network is fed. The tests were conducted using the KDD Cup'99 dataset. It lowers the amount of time needed by algorithms during the training and testing stages, according to experiments. The main drawback of the U2R and R2L attack classes is that they have lower detection rates than other attack classes. Zhang et al. [18] developed a CNN and gcForest-based multilayer complicated IDS model. The P-Zigzag method was also suggested for transforming twodimensional raw data into greyscale pictures. They utilised a coarse grain layer with an enhanced CNN model (GoogLeNetNP) for initial detection. gcForest is then utilised in the fine-grained layer to further categorise the anomalous classes into $\mathrm{N}-1$ subclasses (caXGBoost). They combined the UNSW-NB15 and CIC-IDS 2017 datasets to produce a new one. When compared to single methods, the experimental findings indicate that the proposed model enhances accuracy and detection rate while reducing FAR. 
Jiang et al. [19] presented an enhanced IDS system based on a deep hierarchical network of convolutional neural networks with bidirectional long short-term memory. (BiLSTM). SMOTE is used to boost minority samples in the class imbalance approach, enabling the model to completely learn the features. The spatial characteristics were extracted using the CNN, while the temporal features were extracted using the BiLTSM. The tests utilised the datasets NSL-KDD and UNSWNB15. In terms of accuracy and detection rate, the suggested approach surpasses prior methods. Although detection rates for minority data classes have increased somewhat, they remain very poor in comparison to other attack types. Because of the complicated structure, training takes longer. Yu et al. [20] developed a model for IDS that is based on Few-shot Learning (FSL), a new deep learning (DL) approach [21]. The aim is to train using just a tiny portion of the dataset's balanced labelled data. The model use the embedding functions DNN and CNN to extract the most essential feature and decrease the dimension. According to tests using the NSL-KDD and UNSW-NB15 datasets, the model is capable of attaining acceptable detection rates for minority attack types. To obtain such amazing results on the considered dataset, the suggested model was merely trained on less than $2 \%$ of the data.

\subsubsection{Long-Short Term Memory}

The recurrent neural network (RNN) architecture is a deep learning version or upgrade of the long shortterm memory (LSTM) architecture. The feedback connections of the LSTM are designed to prevent longterm dependence. It can handle whole knowledge sequences as well as single data pieces. For example, LSTMs may be used to accomplish tasks like linked pattern recognition and anomaly detection in network traffic or intrusion detection systems (IDS) (intrusion detection systems). Because there are often unexplained delays between important events, LSTM networks are helpful for categorising, analysing, and generating predictions based on time series data. Vanishing gradients may arise when conventional RNNs are trained. To solve this problem, LSTMs were developed. The insensitivity of LSTMs to gap length is an advantage over RNNs, hidden Markov models, and other sequence learning methods non many situations. According to the researcher, a DLSTM-based architecture in combination with a filterbased feature selection mechanism was described in article [17] for the categorization of different threats in wireless networks. To generate a feature vector that is both minimal and optimum, information gain filtering was employed (IG). A vector with 18 main characteristics was generated as a consequence of this process. The NSL-KDD intrusion detection dataset was utilised in this research. To compare its performance to that of other machine learning and deep learning methods, researchers utilised Artificial Neural Networks and Support Vector Machines. The assessment was based on the F1 score, validation accuracy, and test accuracy. With an accuracy of 86.99 percent and an F1-score of 99.43 percent, the DLSTM IDS was found to be the superior option for multiclass classification. Furthermore, the results of this study were better to those of earlier research [18]. Feed-forward neural networks and the IG filtering method were employed instead of LSTM units in that research. An sophisticated network anomaly detection system was created using deep auto-encoders, deep convolutional neural networks (DCNN), and LSTM in [16]. In an IDS model based on LSTMs, a total of 42 features were applied to 32 LSTM nodes linked to a double-layered feed-forward neural network of ten and one nodes, respectively. The first layer was activated using the Rectified Linear Unit (ReLU) function, while the second layer was activated 
using the sigmoid function. In the experiments, the NSL-KDD dataset was utilised. According to the results, the LSTM IDS presented in this article beat the DCNNIDS in terms of predicted accuracy. On the other hand, the DCNN learnt more quickly. This article [19] proposes a deep learning method for identifying network intrusions. In this approach, convolutional neural networks (CNN) and LSTM models are linked. The NSL-KDD benchmark dataset was used to evaluate the performance of the proposed approach. In this research, the model was compared against a basic recurrent neural network (RNN) and a simple LSTM. The findings revealed that when CNN and LSTM were coupled, they outperformed the other algorithms. To solve the problem of unequal positive and negative learning samples, Jin Yang et al [20] suggested utilising deep convolutional generative adversarial networks (DCGAN), which extracts features directly from raw data and then creates fresh training sets from the raw data. In this article, we utilise long short-term memory (LSTM) to automatically learn the features of network intrusion behaviours. We propose a paradigm based on basic recurrent units to reduce this dependence and allow real-time intrusion detection (SRUs). The suggested model in this research was verified via extensive testing on the industry-standard intrusion detection datasets KDD'99 and NSL-KDD, showing that it efficiently differentiates between normal and malicious network traffic. It is 99.73 percent accurate on the KDD'99 dataset and 99.62 percent accurate on the NSL-KDD dataset. Nikolov et al. examine the impact of a problem-based learning project on a high school student attending a Technology school. [21]. The intrusion detection system employs a recurrent neural network classifier, or more specifically, long-short term memory units. The intrusion detection system is made up of three modules: monitoring, processing, and learning (IDS). The learning module creates the LSTM recurrent neural network by specifying its structure, weights, and biases. For learning, a dataset of system call sequences is utilised.

\subsubsection{Convolution Neural Network - Long Short Term Memory}

In the CNN LSTM architecture, CNN layers for feature extraction from input data are coupled with LSTMs for sequence prediction. CNN LSTMs were developed to address the challenges of visual time series prediction and textual description extraction from picture sequences (e.g. videos). On the front end, CNN layers are utilised, with LSTM and a dense layer at the model's output. This architecture includes the CNN model for feature extraction and the LSTM model for feature interpretation over time steps.

Without changing any hyperparameters, Ahsan et al. [22] developed a hybrid method combining Convolutional Neural Networks (CNN) and Long Short Term Memory (LSTM) that obtained the greatest known accuracy of 99.70 percent on NSLKDD. The convolutional layer was modelled using the Relu function for 100 epochs, whereas the activation layer was simulated with Softmax. The most accurate results of the algorithms were plotted using the Receiver Operating Characteristics (ROC) curve. The author experimented with various kernel sizes and pooling lengths to obtain the best results. Hsu et al [19] presented two deep learning models for intrusion detection systems: LSTM-only and a mix of convolutional neural networks and LSTM (CNN-LSTM). Both approaches outperform the established method, which is based on recurrent neural networks (RNN) and evaluated on the NSL-KDD dataset. Before employing LSTM, a collection of robust features was obtained using convolutional neural networks. Two convolutional layers, each with 32 kernel filters, follow a max pooling layer. On its own, the 
LSTM model comprises two layers and 640 hidden nodes. For each of the LSTM layers, the forget bias is set to one. After training, CNN-LSTM increases its accuracy, scoring 94.12 percent in category-2 and 88.95 percent in category- 5 .

\subsubsection{Gated Recurrent Unit Neural Network}

The gated recurrent unit (GRU) [23], the most commonly used version of LSTM, replaces the three gates in LSTM with a reset gate and an update gate, simplifying the gated structure of the cell. The reset gate controls how the new data input is merged with the prior memory, while the update gate controls how much of the previous memory is stored to the current time step. In terms of performance, GRU has proven to be similar to LSTM and has the potential to save more training time and computing resources.

Yan et al [24] developed LA-GRU, a learning and tuning method based on manually processed real-world network traffic statistics. On the NSL-KDD dataset, the experimental results showed a low false alarm rate while also successfully addressing the learning issue of unbalanced traffic distribution. The LA-GRU model is capable of detecting both high-frequency and low-frequency assaults, with detection rates of 98.34 percent for R2L and 98.61 percent for U2R, respectively. Xu et al [25] examined gated recurrent units (GRU), multilayer perceptron (MLP), and softmax module on the KDD 99 and NSL-KDD datasets. GRU is a superior RNN memory unit for intrusion detection than long short term memory, according to the findings of the experiments (LSTM). On KDD 99 and NSL-KDD, overall detection rates were 99.42 percent and 99.31 percent, respectively, with false positive rates of 0.05 percent and 0.84 percent, respectively. On KDD 99, DOS assaults were detected $99.98 \%$ of the time, but just $99.55 \%$ of the time on NSL-KDD. In the KDD 99 dataset, the proportions of U2R and R2L were 0.01 percent and 0.23 percent, respectively. The proportions of U2R and R2L have been raised to 0.04 percent and 0.79 percent, respectively, in the NSLKDD dataset, which is an updated version of KDD 99. The researchers noted that the system detailed in the article had not been optimised, and that additional research was needed before applying their approach to real-world network traffic. Wang et al. [26] present a novel network intrusion detection model (Conv1d-GRU) for multi-class intrusion detection scenarios that integrates one-dimensional convolution and GRU and corrects for data imbalance by weighting the samples of each category in NSL-KDD. By balancing the sample and loss functions, you may address the issue of data imbalance. With multiclass classification accuracy of $81.65 \%$ and 65.94 percent for the KDDTest + and KDDTest-21 test sets, respectively, the Conv1d-GRU intrusion detection model surpasses its predecessor. Clustering refutes the RNN hypothesis by showing that data properties are connected and that advanced features may be extracted using a mix of one-dimensional convolution and GRU. Kasongo and his associates A Deep Gated Recurrent Unit (DGRU)-based classifier and a wrapper-based feature extraction method for Wireless IDS were created using the NSL-KDD benchmark dataset. A DGRU RNN is used in combination with a Multilayer Perceptron (MLP). For the purposes of the experiment, a reduced input feature vector of 18 items was generated. Validation accuracy increased to 99.35 percent, F-Measure accuracy to 99.67 percent, and test accuracy to 88.42 percent as a result of the experiments. Ahsan el al. [22] explored the application of LSTM and GRU-based deep learning algorithms for intrusion detection. The aim was to use accuracy and F1-score performance criteria to assess the effectiveness of these models. Both the BGP 
and the well-known NSL-KDD datasets were utilised in the research. Several LSTM and GRU models were implemented, with particular emphasis paid to the NSL-KDD. The top performing LSTM IDS model achieved an accuracy of 82.78 percent and an F1-score of 83.34 percent when applied to test data. The GRUIDS had an F1-score of 83.05 percent and an accuracy of 82.87 percent on test data.

\section{Iv. Evaluation Metrics}

\subsection{Dataset}

The NSL-KDD datasets have been refined from the KDDCup'99 datasets. It clears the KDD Cup'99 of extraneous records in order to get impartial classification results. By removing duplicated data, a better detection rate may be obtained. The NSL-KDD dataset consists of two parts: KDDTrain+, a full training dataset in CSV format marked with attack types and difficulty levels, and KDDTest+, a complete testing dataset in CSV format annotated with attack types and difficulty levels. At random, three 50,000-record subsets of the KDD training set were created in the manner described in [28]. They utilised 21 learning machines that had been trained on the built training sets to label the records of the complete training and testing sets. As a consequence of this experiment, each record had 21 projected labels. The difficulty level of the NSL-KDD dataset is determined by the number of successful prediction values generated by each of the 21 learning machines. The successful prediction value is the number of learning machines that successfully predicted the label. The collection's records have a maximum difficulty level of 21 . The specifics of the different classes in the dataset are shown in Tables 2 and 3.

The four simulated attacks like [29][30]

1. Denial of Service Attack (DoS): This attack tries to "shut down a network, computer, or process; or otherwise denies approved users the use of assets or services.

2. User to Root Attack (U2R): The attacker has an account on a system and can misuse the user rights by exploiting the vulnerabilities in a system. An attacker with higher privileges attempting to become root or user.

3. Remote to Local Attack (R2L) : R2L occurs when an attacker sends packets over a network to a machine but is unable to gain local access by exploiting a vulnerability as a user of that machine.

4. Probing Attack: It is an attempt to collect data about a computer network used to attack chosen machines and services. 
Table 2

NSL-KDD attack types by total number of instances

\begin{tabular}{|c|c|c|c|}
\hline Attack & $\begin{array}{l}\text { Total Instances in } \\
\text { NSL-KDD Dataset }\end{array}$ & $\begin{array}{l}\text { Attack } \\
\text { Category }\end{array}$ & Description \\
\hline $\begin{array}{l}\text { Buffer } \\
\text { overflow }\end{array}$ & 30 & \multirow[t]{4}{*}{ U2R } & \multirow[t]{4}{*}{$\begin{array}{l}\text { Acquiring root or super-user access to a } \\
\text { computer }\end{array}$} \\
\hline Loadmodule & 9 & & \\
\hline perl & 3 & & \\
\hline rootkit & 2931 & & \\
\hline $\begin{array}{l}\text { guess- } \\
\text { passwd }\end{array}$ & 53 & \multirow[t]{8}{*}{ R2L } & \multirow[t]{8}{*}{ Unauthorized access from a remote computer } \\
\hline ftp-write & 8 & & \\
\hline Imap & 11 & & \\
\hline Phf & 4 & & \\
\hline Multihop & 7 & & \\
\hline Warezmaster & 20 & & \\
\hline Warezclient & 1020 & & \\
\hline Spy & 2 & & \\
\hline Ipsweep & 3599 & \multirow[t]{3}{*}{ Probe } & \multirow{3}{*}{$\begin{array}{l}\text { The attacker's objective is to exhaust network } \\
\text { resources. }\end{array}$} \\
\hline Nmap & 1493 & & \\
\hline Portsweep & 2931 & & \\
\hline Back & 956 & \multirow[t]{7}{*}{ Dos } & \multirow{7}{*}{$\begin{array}{l}\text { The attacker's objective is to degrade the } \\
\text { performance of network resources. }\end{array}$} \\
\hline Land & 18 & & \\
\hline Neptune & 41,214 & & \\
\hline Pod & 201 & & \\
\hline Smurf & 2646 & & \\
\hline Teardrop & 892 & & \\
\hline Satan & 3633 & & \\
\hline Normal & 67,343 & Normal & Typical connection logs \\
\hline
\end{tabular}


Table 3

NSL-KDD distribution of normal and attack records.

\begin{tabular}{|lllllll|}
\hline Dataset & R2L & U2R & Probe & DoS & Normal & Instances \\
\hline KDD Train+ & 995 & 52 & 11656 & 45927 & 67343 & 125973 \\
\hline KDD Test+ & 2885 & 67 & 2421 & 7460 & 9711 & 22544 \\
\hline
\end{tabular}

\subsection{Statistical Measures}

The most popular Key performance indicators for assessing IDS's machine learning and deep learning algorithms' performance. All evaluation criteria are built on the confusion Matrix, a two-dimensional matrix that contains information about the Actual and Predicted classes.

i. True-Positive (TP): Data instances that the classifier correctly predicts as an Attack.

ii. False-Negative (FN): Data instances that were predicted incorrectly as Normal.

iii. False-Positive (FP): Instances of data that were identified mistakenly as an Attack.

iv. True-Negative (TN): Cases that were classified accurately as Normal.

The diagonal members of the confusion matrix indicate accurate predictions, whereas the nondiagonal components represent incorrect assumptions produced by a certain classifier. These features of the confusion matrix are shown in Table 4. In addition, the following are some of the numerous assessment measures used in recent studies:

Table 4

Confusion matrix

\begin{tabular}{|llll|}
\hline \multirow{3}{*}{ Actual Class } & \multicolumn{3}{c|}{ Predicted Class } \\
\cline { 2 - 4 } & & Attack & Normal \\
\cline { 2 - 4 } & Attack & True Positive & False Negative \\
\cline { 2 - 4 } & Normal & False Positive & True Negative \\
\hline
\end{tabular}

On the basis of the aforementioned terms, the following most often used evaluation measures are evaluated.

\subsubsection{Accuracy}


It computes the percentage of successfully recognised connection records in comparison to the entire test dataset. The accuracy of the machine learning model is better if it is greater (Accuracy $\in[0 ; 1]$ ). Accuracy is a useful metric to use while evaluating the test dataset, which contains balanced classes and is defined as follows:

Accuracy $=\frac{T P+T N}{T P+F P+T N+F N}$

\subsubsection{Precision}

The ratio of accurately detected attack connection records to all identified attack connection records is calculated. The machine learning model performs better with a higher Precision (Precision $\in[0 ; 1])$. The term "precision" means the following:

Precision $=\frac{T P}{T P+F P}$

\subsubsection{F1-Measure}

F1-Measure is a subset of F1-Score. Precision and Recall have a synergistic relationship. The F1-Score of the machine learning model is better if it is greater (F1 - Score $\in[0 ; 1]$ ). The following is the F1-score:

F1- Score $=2 * \frac{\text { Precision } * \text { Recall }}{\text { Precision }+ \text { Recall }}$

\subsubsection{Recall}

Recall is the ratio of all samples categorised correctly as Attacks to all samples classified as Attacks. Additionally, it is referred to as a Detection Rate. The machine learning model performs better when the Recall value is greater than zero (Recall $\in[0 ; 1]$ ). Additionally, it is referred to as the True Positive Rate. The term recall has the following definition:

Recall/TPR $=\frac{T P}{T P+F N}$

\subsubsection{False Positive Rate (FPR)}

It determines the percentage of Normal connection records that have been labelled as Attacks in comparison to the total amount of Normal connection records. When the FPR is less than one (FPR $\in[0$; 1]), the machine learning model performs better. FPR is defined as follows:

$\mathrm{FPR}=\frac{F P}{F P+T N}$

\subsubsection{Receiver Operating Characteristics (ROC) curve}

The trade-off between the TPR on the $y$ axis and the FPR on the $x$ axis at various thresholds is plotted on the receiver operating characteristic $(\mathrm{ROC})$ curve. The area beneath the receiver operating characteristic 
curve (AUC), which is used in conjunction with the ROC as a comparative statistic for machine learning models, is the area beneath the receiver operating characteristic curve. The AUC is a metric that indicates how well a machine learning model performs.

\section{Results}

\subsection{System Configuration}

All studies were conducted on a Windows 10 operating system with Python Anaconda. Deep Learning Algorithms were built using TensorFlow as the backend and the Keras higher level framework as the frontend. The CPU was configured as follows: (16GB RAM, 2TB hard drive, Intel(R) core(TM) i5-4200U CPU@1.60GHz).

\subsection{Steps Involved in Model Development}

The parameters of deep learning algorithms have an effect on classification performance (number of hidden nodes, multiple hidden layers, and optimization algorithms). The experimental procedure is as follows:

- Step 1: Normalize the training and test datasets.

- Step 2: Using a range of layers and validation data, train the deep learning models. Fine-tune the parameters of the deep learning models.

- Step 3: Validate the deep learning models.

- Step 4: Evaluate the models' accuracy.

The following Fig. 3 illustrates the process of developing and testing deep learning models.

\subsection{Performance Evaluation}

To establish a baseline method, we used NSLKDD datasets to test the performance of deep learning model. The L2 method was used to divide the datasets into train and test subsets and normalise them and determining the model's accuracy.

Below Tables 5, 6, 7, 8, 9 denotes the comparison of proposed model development with traditional model. 
Table 5

Details about the structure proposed CNN model with Traditional CNN Model

\begin{tabular}{|c|c|c|c|c|c|}
\hline \multicolumn{3}{|c|}{ CNN Traditional Model } & \multicolumn{3}{|c|}{ CNN (Proposed Model) } \\
\hline Layer type & Output Shape & Parameters \# & Layer type & Output Shape & Parameters \# \\
\hline Conv1D & (None, 41, 64) & 256 & Conv1D & (None, 41, 64) & 256 \\
\hline Max Pooling & (None, 20, 64) & 0 & Conv1D & (None, 41, 64) & 12352 \\
\hline Flatten & (None, 1280) & 0 & Max Pooling & (None, 20, 64) & 0 \\
\hline Dense & (None, 128) & 163968 & Conv1D & (None, 20, 128) & 24704 \\
\hline Dropout & (None, 128) & 0 & Conv1D & (None, 20, 128) & 49280 \\
\hline \multirow[t]{5}{*}{ Dense } & (None, 1) & 129 & Max Pooling & (None, 10, 128) & 0 \\
\hline & & & Flatten & (None, 1280) & 0 \\
\hline & & & Dense & (None, 128) & 163968 \\
\hline & & & Dropout & (None, 128) & 0 \\
\hline & & & Dense & (None, 1) & 129 \\
\hline \multicolumn{3}{|c|}{ Total params: 164,353} & \multicolumn{3}{|c|}{ Total params: 250,689} \\
\hline \multicolumn{3}{|c|}{ Trainable params: 164,353} & \multicolumn{3}{|c|}{ Trainable params: 250,689} \\
\hline \multicolumn{3}{|c|}{ Non-trainable params: 0} & \multicolumn{3}{|c|}{ Non-trainable params: 0} \\
\hline
\end{tabular}


Table 6

Details about the structure proposed CNN LSTM model with Traditional CNN LSTM Model

\begin{tabular}{|c|c|c|c|c|c|}
\hline \multicolumn{3}{|c|}{ CNN LSTM Traditional Model } & \multicolumn{3}{|c|}{ CNN LSTM (Proposed Model) } \\
\hline Layer type & Output Shape & Parameters \# & Layer type & Output Shape & Parameters \# \\
\hline Conv1D & (None, 41, 64) & 256 & Conv1D & (None, 41, 64) & 256 \\
\hline Max Pooling & (None, 20, 64) & 0 & Conv1D & (None, 41, 64) & 123 \\
\hline LSTM & (None, 70) & 378 & Max Pooling & (None, 20, 64) & 0 \\
\hline Dropout & (None, 70) & 0 & Conv1D & (None, 20, 128) & 247 \\
\hline \multirow[t]{5}{*}{ Dense } & (None, 1) & 71 & Conv1D & (None, 20, 128) & 492 \\
\hline & & & Max Pooling & (None, 10, 128) & 0 \\
\hline & & & LSTM & (None, 70) & 557 \\
\hline & & & Dropout & (None, 70) & 0 \\
\hline & & & Dense & (None, 1) & 71 \\
\hline \multicolumn{3}{|c|}{ Total params: 38,127} & \multicolumn{3}{|c|}{ Total params: 142,383} \\
\hline \multicolumn{3}{|c|}{ Trainable params: 38,127} & \multicolumn{3}{|c|}{ Trainable params: 142,383} \\
\hline \multicolumn{3}{|c|}{ Non-trainable params: 0} & \multicolumn{3}{|c|}{ Non-trainable params: 0} \\
\hline
\end{tabular}


Table 7

Details about the structure proposed RNN model with Traditional RNN Model

\begin{tabular}{|c|c|c|c|c|c|}
\hline \multicolumn{3}{|c|}{ RNN (Traditional Model) } & \multicolumn{3}{|c|}{ RNN (Proposed Model) } \\
\hline Layer type & Output Shape & Parameters \# & Layer type & Output Shape & Parameters \# \\
\hline Simple RNN & (None, 41, 8) & 80 & Simple RNN & (None, 41, 32) & 108 \\
\hline Dropout & (None, 41, 8) & 0 & Dropout & (None, 41, 32) & 0 \\
\hline Simple RNN & (None, 8) & 136 & Simple RNN & (None, 41, 32) & 208 \\
\hline Dropout & (None, 8) & 0 & Dropout & (None, 41, 32) & 0 \\
\hline Dense & (None, 1) & 9 & Simple RNN & (None, 41, 32) & 208 \\
\hline \multirow[t]{5}{*}{ Activation } & (None, 1) & 0 & Dropout & (None, 41, 32) & 0 \\
\hline & & & Simple RNN & (None, 32) & 208 \\
\hline & & & Dropout & (None, 32) & 0 \\
\hline & & & Dense & (None, 1) & 33 \\
\hline & & & Activation & (None, 1) & 0 \\
\hline \multicolumn{3}{|c|}{ Total params: 225} & \multicolumn{3}{|c|}{ Total params: 142,383} \\
\hline \multicolumn{3}{|c|}{ Trainable params: 225} & \multicolumn{3}{|c|}{ Trainable params: 142,383} \\
\hline \multicolumn{3}{|c|}{ Non-trainable params: 0} & \multicolumn{3}{|c|}{ Non-trainable params: 0} \\
\hline
\end{tabular}


Table 8

Details about the structure proposed LSTM model with Traditional LSTM Model

\begin{tabular}{|c|c|c|c|c|c|}
\hline Layer type & Output Shape & Parameters \# & Layer type & Output Shape & Parameters \# \\
\hline LSTM & (None, 41, 8) & 320 & LSTM & (None, 41, 32) & 435 \\
\hline Dropout & (None, 41, 8) & 0 & Dropout & (None, 41, 32) & 0 \\
\hline LSTM & (None, 8) & 544 & LSTM & (None, 41, 32) & 832 \\
\hline Dropout & (None, 8) & 0 & Dropout & (None, 41, 32) & 0 \\
\hline Dense & (None, 1) & 9 & LSTM & (None, 41, 32) & 832 \\
\hline \multirow[t]{5}{*}{ Activation } & (None, 1) & 0 & Dropout & (None, 41, 32) & 0 \\
\hline & & & LSTM & (None, 32) & 832 \\
\hline & & & Dropout & (None, 32) & 0 \\
\hline & & & Dense & (None, 1) & 33 \\
\hline & & & Activation & (None, 1) & 0 \\
\hline \multicolumn{3}{|c|}{ Total params: 873} & \multicolumn{3}{|c|}{ Total params: 29,345} \\
\hline \multicolumn{3}{|c|}{ Trainable params: 873} & \multicolumn{3}{|c|}{ Trainable params: 29,345} \\
\hline \multicolumn{3}{|c|}{ Non-trainable params: 0} & \multicolumn{3}{|c|}{ Non-trainable params: 0} \\
\hline
\end{tabular}


Table 9

Details about the structure proposed GRU model with Traditional GRU Model

\begin{tabular}{|c|c|c|c|c|c|}
\hline Layer type & Output Shape & Parameters \# & Layer type & Output Shape & Parameters \# \\
\hline GRU & (None, 41, 8) & 240 & GRU & (None, 41, 32) & 326 \\
\hline Dropout & (None, 41, 8) & 0 & Dropout & (None, 41, 32) & 0 \\
\hline GRU & (None, 8) & 408 & GRU & (None, 41, 32) & 624 \\
\hline Dropout & (None, 8) & 0 & Dropout & (None, 41,32) & 0 \\
\hline Dense & (None, 1) & 9 & GRU & (None, 41, 32) & 624 \\
\hline \multirow[t]{5}{*}{ Activation } & (None, 1) & 0 & Dropout & (None, 41, 32) & 0 \\
\hline & & & GRU & (None, 32) & 624 \\
\hline & & & Dropout & (None, 32) & 0 \\
\hline & & & Dense & (None, 1) & 33 \\
\hline & & & Activation & (None, 1) & 0 \\
\hline \multicolumn{3}{|c|}{ Total params: 657} & \multicolumn{3}{|c|}{ Total params: 22,017} \\
\hline \multicolumn{3}{|c|}{ Trainable params: 657} & \multicolumn{3}{|c|}{ Trainable params: 22,017} \\
\hline \multicolumn{3}{|c|}{ Non-trainable params: 0} & \multicolumn{3}{|c|}{ Non-trainable params: 0} \\
\hline
\end{tabular}


Table 10

Denote Test results of deep learning algorithm results for Binary class classification.

\begin{tabular}{|lllll|}
\hline Model & Accuracy & Precision & Recall & F-score \\
\hline CNN (Traditional) & 0.993948 & 0.999554 & 0.990119 & 0.999117 \\
\hline CNN (Proposed) & $\mathbf{0 . 9 9 4 2 5 6}$ & $\mathbf{1}$ & $\mathbf{0 . 9 8 9 8 7 2}$ & $\mathbf{0 . 9 9 9 5 5 9}$ \\
\hline CNN-LSTM (Traditional) & 0.986969 & 0.988082 & 0.983202 & 0.995146 \\
\hline CNN (Proposed) & $\mathbf{0 . 9 9 1 8 4 8}$ & $\mathbf{0 . 9 9 4 4 3 1}$ & $\mathbf{0 . 9 8 8 6 3 6}$ & $\mathbf{0 . 9 9 7 3 5 2}$ \\
\hline RNN (Traditional) & 0.98913 & 0.989084 & 0.988636 & 0.997793 \\
\hline RNN (Proposed) & $\mathbf{0 . 9 8 9 9 1}$ & $\mathbf{0 . 9 9 0 1 9 8}$ & $\mathbf{0 . 9 8 7 4 0 1}$ & $\mathbf{0 . 9 9 7 7 9 3}$ \\
\hline LSTM (Traditional) & 0.985301 & 0.986968 & 0.984684 & 0.991615 \\
\hline LSTM (Proposed) & $\mathbf{0 . 9 8 8 7 6}$ & $\mathbf{0 . 9 8 5 1 8 6}$ & $\mathbf{0 . 9 8 7 1 5 4}$ & $\mathbf{0 . 9 8 8 5 2 6}$ \\
\hline GRU (Traditional) & 0.985672 & 0.976387 & 0.982708 & 0.986761 \\
\hline GRU (Proposed) & $\mathbf{0 . 9 9 1 7 8 6}$ & $\mathbf{0 . 9 9 3 7 6 3}$ & $\mathbf{0 . 9 8 9 8 7 2}$ & $\mathbf{0 . 9 9 7 3 5 2}$ \\
\hline
\end{tabular}

\section{Vi. Conclusion}

In this study, we propose from the NSL KDD data to train models using Deep Convolutional Neural Network (DCNN), Deep Recurrent Neural Network (DRNN), Deep Long Short-Term Memory (DLSTM), Deep Convolutional Neural Network-Long Short-Term Memory (DCNNLSTM), and Deep Gated Recurrent Unit (DGRU). As the model's layer count increases, our proposed models show excellent accuracy. In general, training complex deep learning architectures on advanced hardware in a distributed fashion can improve performance significantly. This work using the benchmark IDS datasets due to the high computational cost of advanced designs. In this environment, it will be a critical task, and it is seen as a critical path for future work.

\section{Vii. Declarations}

Funding: No funds, grants, or other support was received.

Conflicts of interest/Competing interests: There is no potential of conflict of Interest between the authors regarding the manuscript preparation and submission.

Availability of data and material: For experimentations purpose we used NSLKDD data set. https://www.unb.ca/cic/datasets/nsl.html

Code availability: For experimentations purpose we used Python and keras both are open source. 


\section{Viii. References}

[1] A. B. Abhale and S. S. Manivannan, "Review on intrusion detection system in wireless sensor network," J. Adv. Res. Dyn. Control Syst., vol. 11, no. 7 Special Issue, pp. 954-971, 2019.

[2] Dr. E. Baraneetharan, "Role of Machine Learning Algorithms Intrusion Detection in WSNs: A Survey," J. Inf. Technol. Digit. World, vol. 02, no. 03, pp. 161-173, 2020.

[3] Ashwini B. Abhale and S. S. Manivannan, "Supervised Machine Learning Classification Algorithmic Approach for Finding Anomaly Type of Intrusion Detection in Wireless Sensor Network," Opt. Mem. Neural Networks, vol. 29, no. 3, pp. 244-256, Jul. 2020.

[4] N. Shone, T. N. Ngoc, V. D. Phai, and Q. Shi, "A Deep Learning Approach to Network Intrusion Detection," IEEE Trans. Emerg. Top. Comput. Intell., vol. 2, no. 1, pp. 41-50, Feb. 2018.

[5] M. Abadi et al., "TensorFlow: Large-Scale Machine Learning on Heterogeneous Distributed Systems," Mar. 2016.

[6] S. Choudhary and N. Kesswani, "Analysis of KDD-Cup'99, NSL-KDD and UNSW-NB15 Datasets using Deep Learning in loT," Procedia Comput. Sci., vol. 167, pp. 1561-1573, 2020.

[7] C. Xu, J. Shen, X. Du, and F. Zhang, "An Intrusion Detection System Using a Deep Neural Network with Gated Recurrent Units," IEEE Access, vol. 6, pp. 48697-48707, 2018.

[8] A. Khraisat, I. Gondal, P. Vamplew, and J. Kamruzzaman, "Survey of intrusion detection systems: techniques, datasets and challenges," Cybersecurity, vol. 2, no. 1, p. 20, Dec. 2019.

[9] A. Agarwal and N. C. Kaushal, "A Study of Intrusion Detection System in Wireless Sensor Network," 2020, pp. 337-343.

[10] M. Labonne, "Anomaly-based network intrusion detection using Maxime Labonne To cite this version: HAL Id: tel-02988296 Anomaly-Based Network Intrusion Detection Using Machine Learning," 2020.

[11] G.-B. Huang, Q.-Y. Zhu, and C.-K. Siew, “Extreme learning machine: Theory and applications,” Neurocomputing, vol. 70, no. 1-3, pp. 489-501, Dec. 2006.

[12] G. Li, P. Niu, X. Duan, and X. Zhang, "Fast learning network: a novel artificial neural network with a fast learning speed," Neural Comput. Appl., vol. 24, no. 7-8, pp. 1683-1695, Jun. 2014.

[13] M. H. Ali, B. A. D. Al Mohammed, A. Ismail, and M. F. Zolkipli, "A New Intrusion Detection System Based on Fast Learning Network and Particle Swarm Optimization," IEEE Access, vol. 6, pp. 2025520261, 2018. 
[14] C. Yin, Y. Zhu, J. Fei, and X. He, "A Deep Learning Approach for Intrusion Detection Using Recurrent Neural Networks," IEEE Access, vol. 5, pp. 21954-21961, 2017.

[15] M. Sheikhan, Z. Jadidi, and A. Farrokhi, "Intrusion detection using reduced-size RNN based on feature grouping," Neural Comput. Appl., vol. 21, no. 6, pp. 1185-1190, Sep. 2012.

[16] S. Naseer et al., "Enhanced Network Anomaly Detection Based on Deep Neural Networks," IEEE Access, vol. 6, pp. 48231-48246, 2018.

[17] S. M. Kasongo and Y. Sun, "A Deep Long Short-Term Memory based classifier for Wireless Intrusion Detection System," ICT Express, vol. 6, no. 2, pp. 98-103, Jun. 2020.

[18] S. M. Kasongo and Y. Sun, "A deep learning method with filter based feature engineering for wireless intrusion detection system," IEEE Access, vol. 7, pp. 38597-38607, 2019.

[19] C.-M. Hsu, H.-Y. Hsieh, S. W. Prakosa, M. Z. Azhari, and J.-S. Leu, "Using Long-Short-Term Memory Based Convolutional Neural Networks for Network Intrusion Detection," 2019, pp. 86-94.

[20] J. Yang, T. Li, G. Liang, W. He, and Y. Zhao, "A Simple Recurrent Unit Model Based Intrusion Detection System With DCGAN," IEEE Access, vol. 7, pp. 83286-83296, 2019.

[21] D. Nikolov, I. Kordev, and S. Stefanova, "Concept for network intrusion detection system based on recurrent neural network classifier," in 2018 IEEE XXVII International Scientific Conference Electronics - ET, 2018, pp. 1-4.

[22] M. Ahsan and K. E. Nygard, "Convolutional neural networks with LSTM for intrusion detection," Epic Ser. Comput., vol. 69, pp. 69-79, 2020.

[23] K. Cho et al., "Learning Phrase Representations using RNN Encoder-Decoder for Statistical Machine Translation," Jun. 2014.

[24] B. Yan and G. Han, "LA-GRU: Building Combined Intrusion Detection Model Based on Imbalanced Learning and Gated Recurrent Unit Neural Network," Secur. Commun. Networks, vol. 2018, pp. 1-13, Aug. 2018.

[25] C. Xu, J. Shen, X. Du, and F. Zhang, "An Intrusion Detection System Using a Deep Neural Network With Gated Recurrent Units," IEEE Access, vol. 6, pp. 48697-48707, 2018.

[26] X. Wang and B. Xiao, "Intrusion detection using a combination of one-dimensional convolution and GRU," J. Phys. Conf. Ser., vol. 1684, p. 012083, Nov. 2020.

[27] S. M. Kasongo and Y. Sun, "A Deep Gated Recurrent Unit based model for wireless intrusion detection system," ICT Express, vol. 7, no. 1, pp. 81-87, Mar. 2021. 
[28] M. Tavallaee, E. Bagheri, W. Lu, and A. A. Ghorbani, "A detailed analysis of the KDD CUP 99 data set," in 2009 IEEE Symposium on Computational Intelligence for Security and Defense Applications, 2009, pp. $1-6$.

[29] L. Dhanabal and S. P. Shantharajah, "A Study on NSL-KDD Dataset for Intrusion Detection System Based on Classification Algorithms," Int. J. Adv. Res. Comput. Commun. Eng., vol. 4, no. 6, pp. 446-452, 2015.

[30] Y. Hamid, V. R. Balasaraswathi, L. Journaux, and M. Sugumaran, "Benchmark Datasets for Network Intrusion Detection: A Review," Int. J. Netw. Secur., vol. 20, no. 4, p. 7, 2018.

\section{Figures}

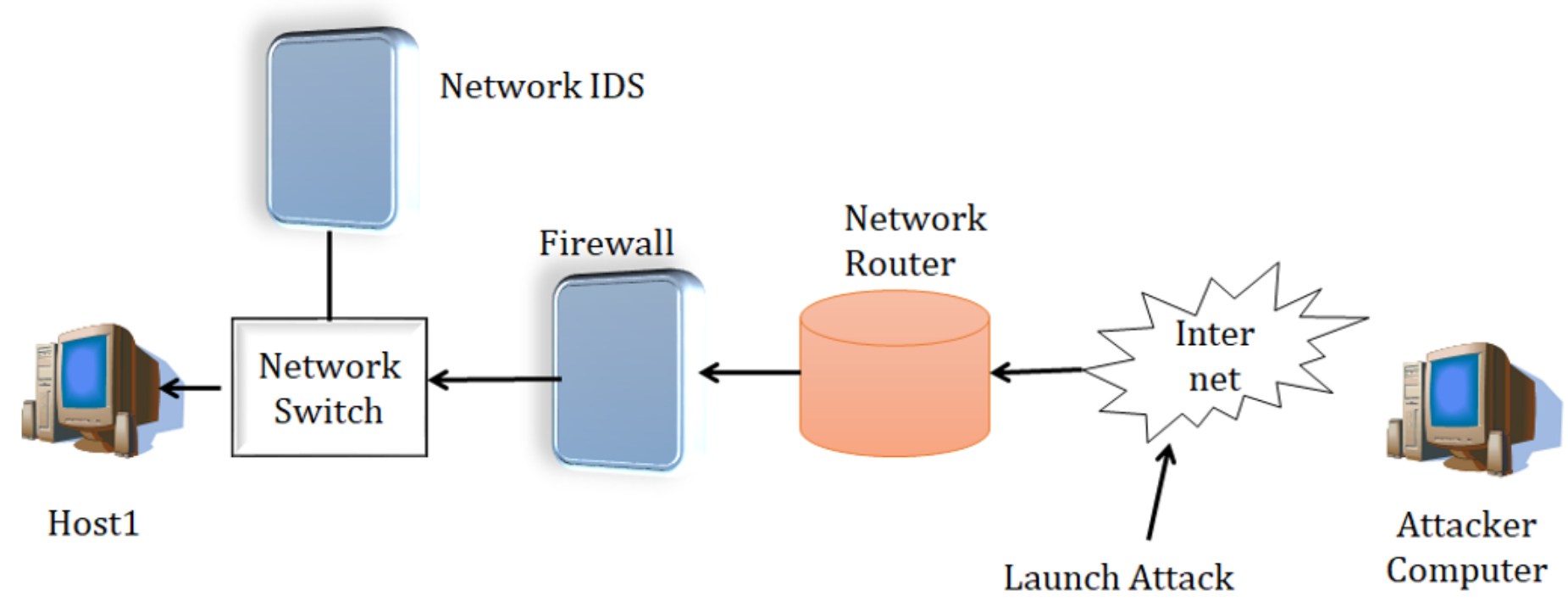

\section{Figure 1}

NIDS deployment on the network 


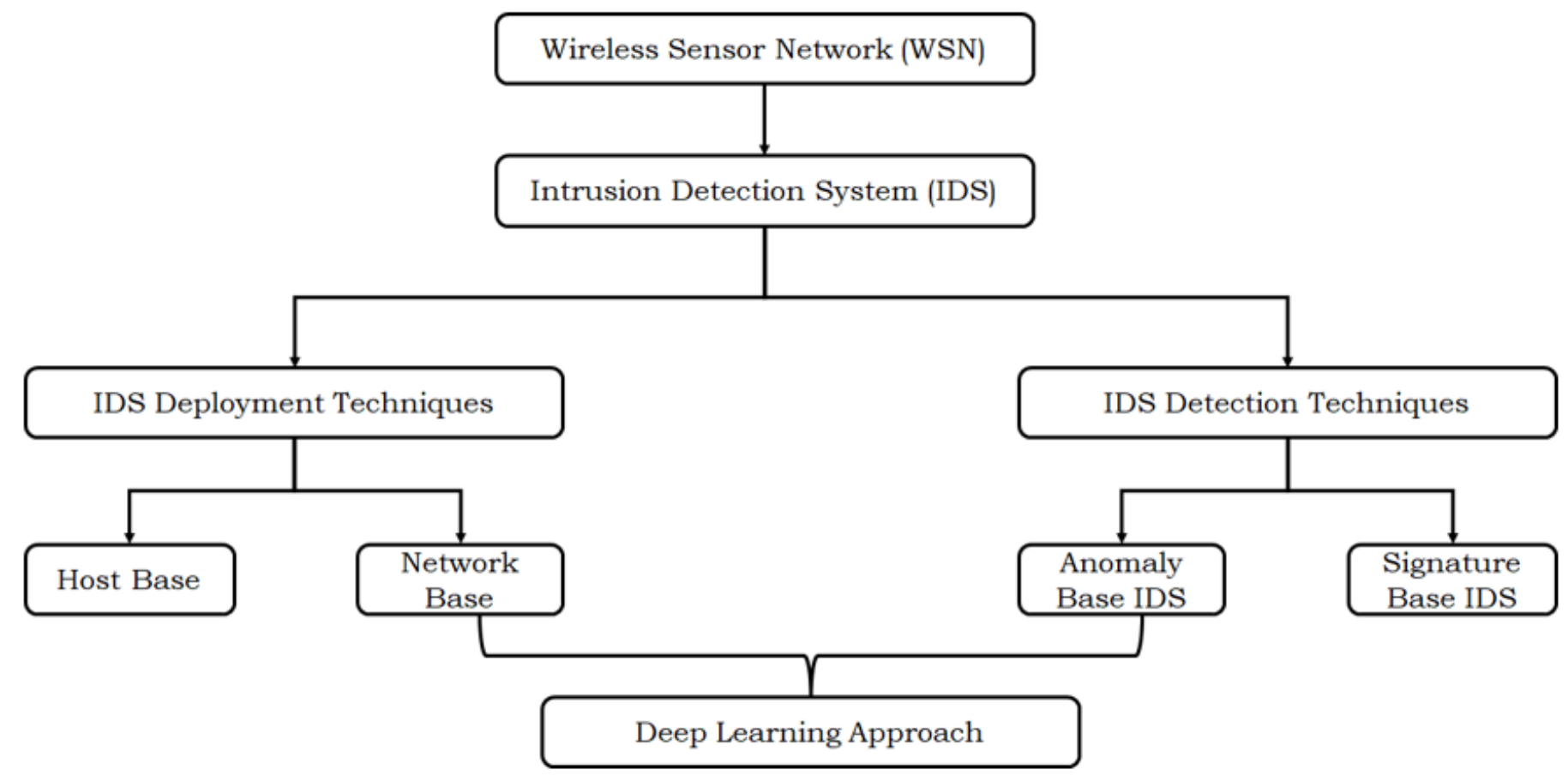

Figure 2

Taxonomy for classification of intrusion detection systems 


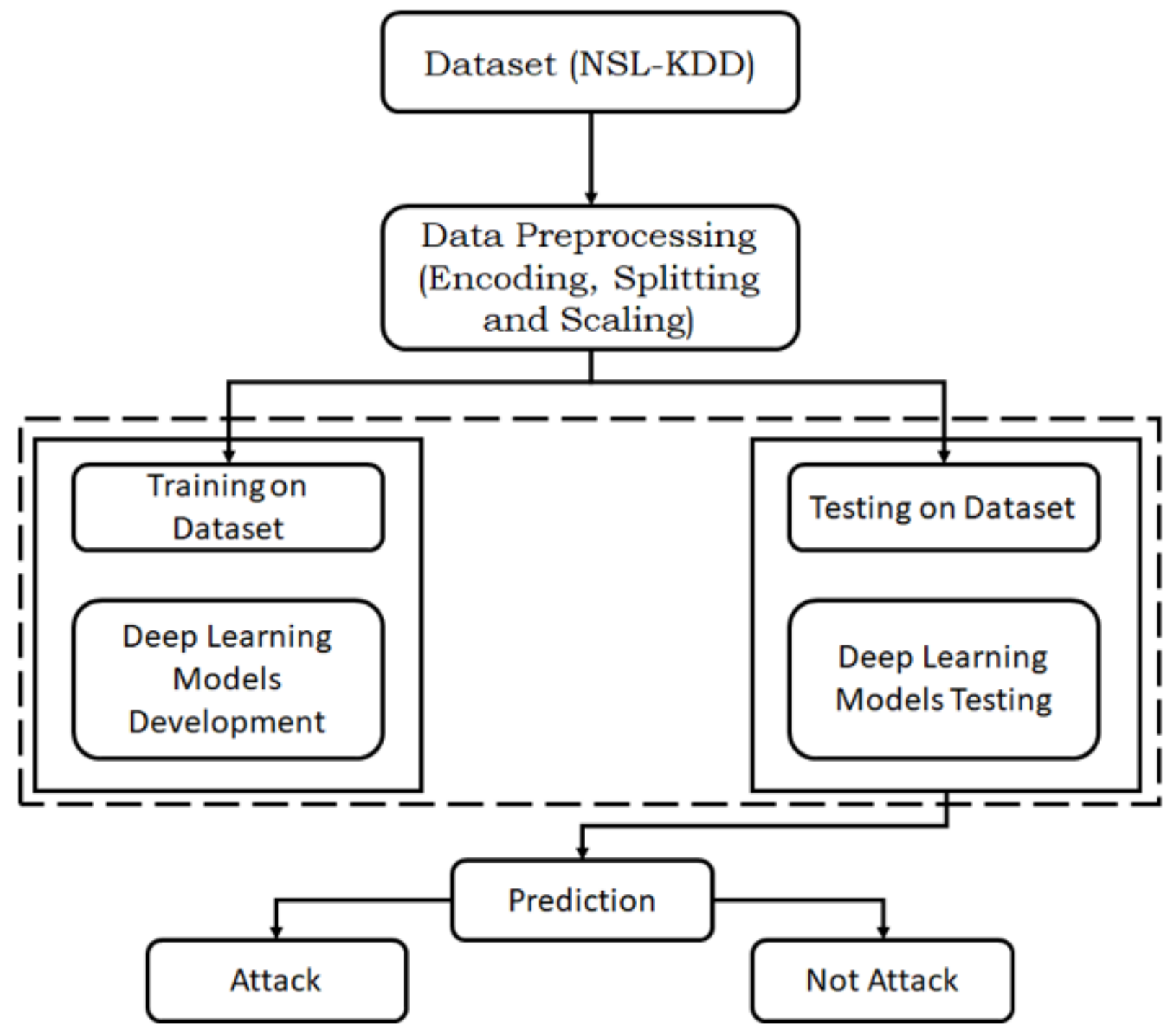

Figure 3

Working of deep learning model development and testing

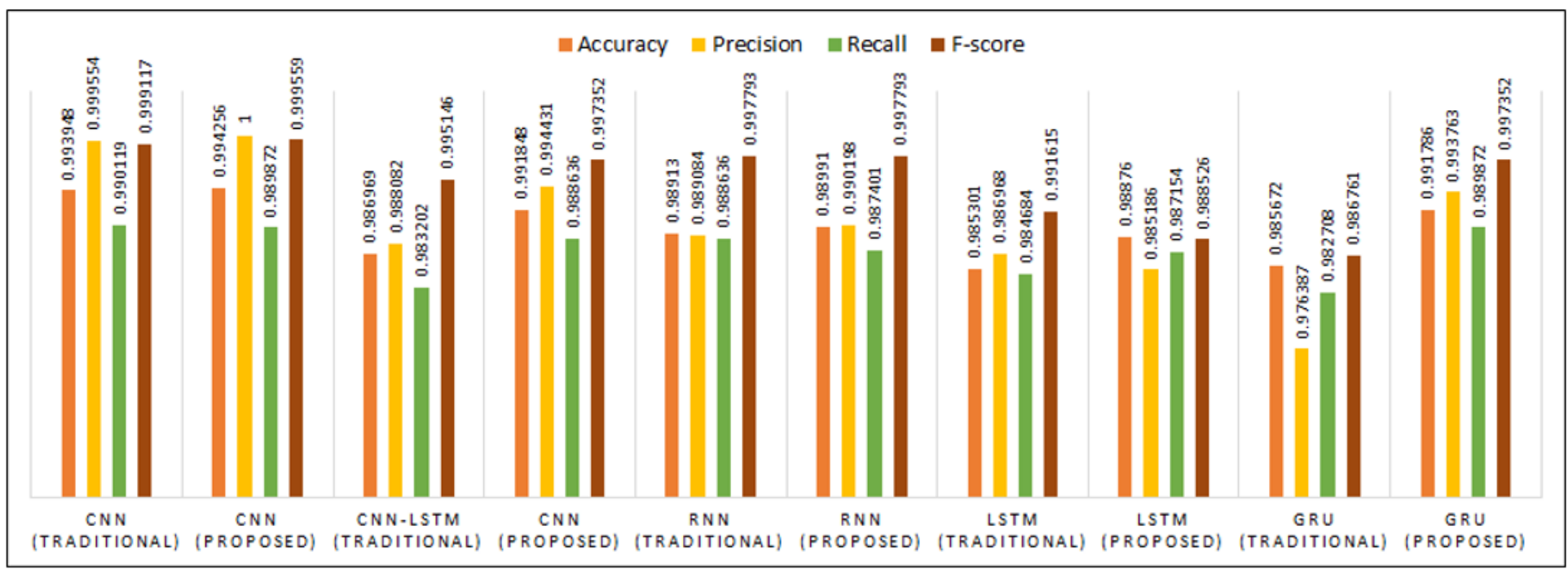


Figure 4

Comparison of all model developed by Accuracy, Precision, Recall and F1 Score.

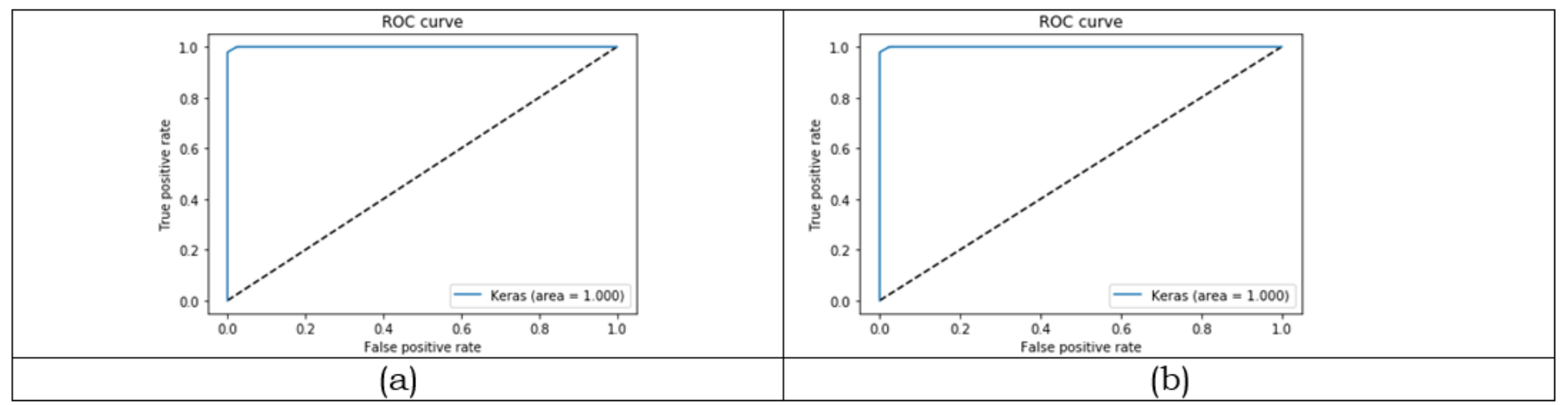

\section{Figure 5}

ROC curves of (a) NSL KDD dataset using CNN (Traditional) (b) NSL KDD dataset using CNN (Proposed)

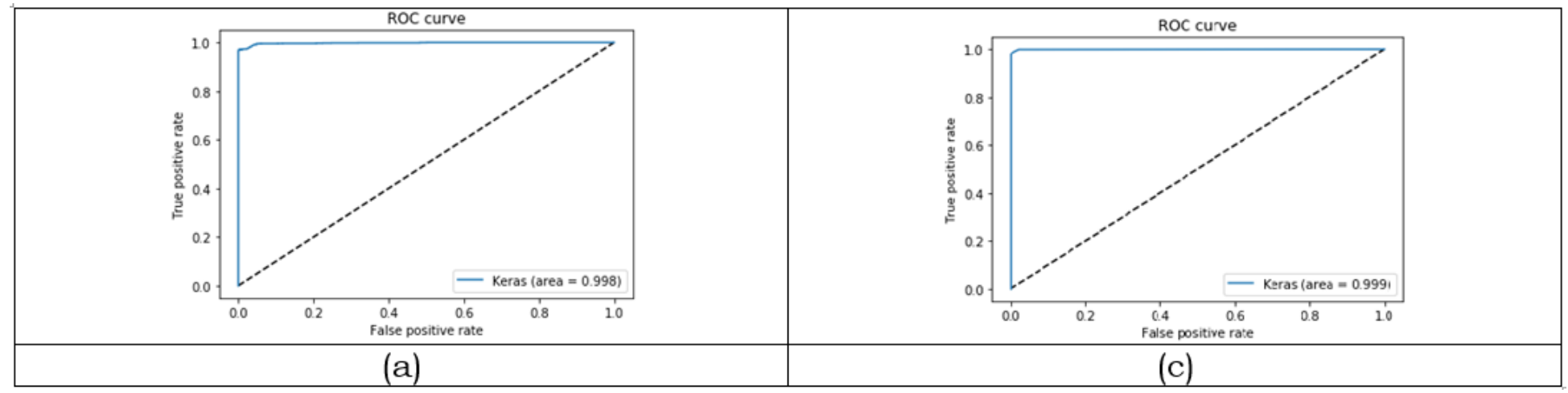

\section{Figure 6}

ROC curves of (a) NSL KDD dataset using CNN-LSTM (Traditional) (b) NSL KDD dataset using CNNLSTM (Proposed)

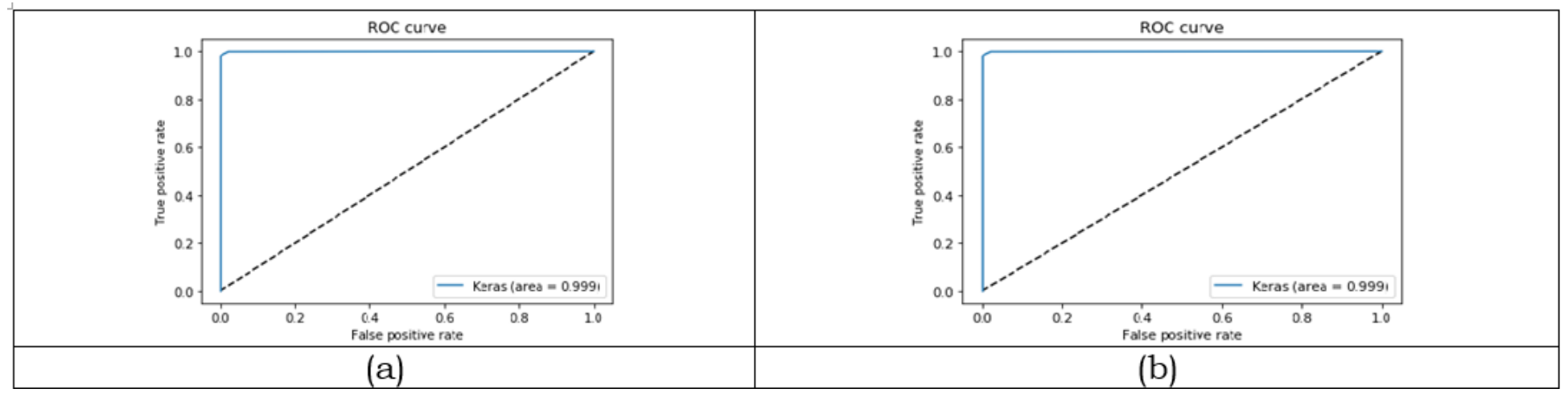

\section{Figure 7}

ROC curves of (a) NSL KDD dataset using RNN (Traditional) (b) NSL KDD dataset using RNN (Proposed) 


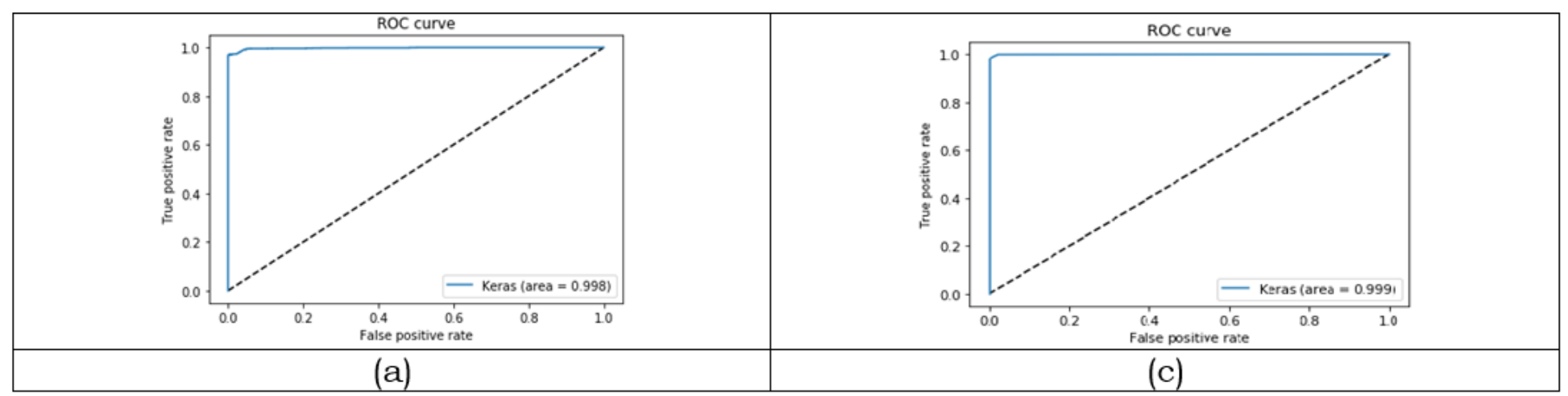

\section{Figure 8}

ROC curves of (a) NSL KDD dataset using LSTM (Traditional) (b) NSL KDD dataset using LSTM (Proposed)

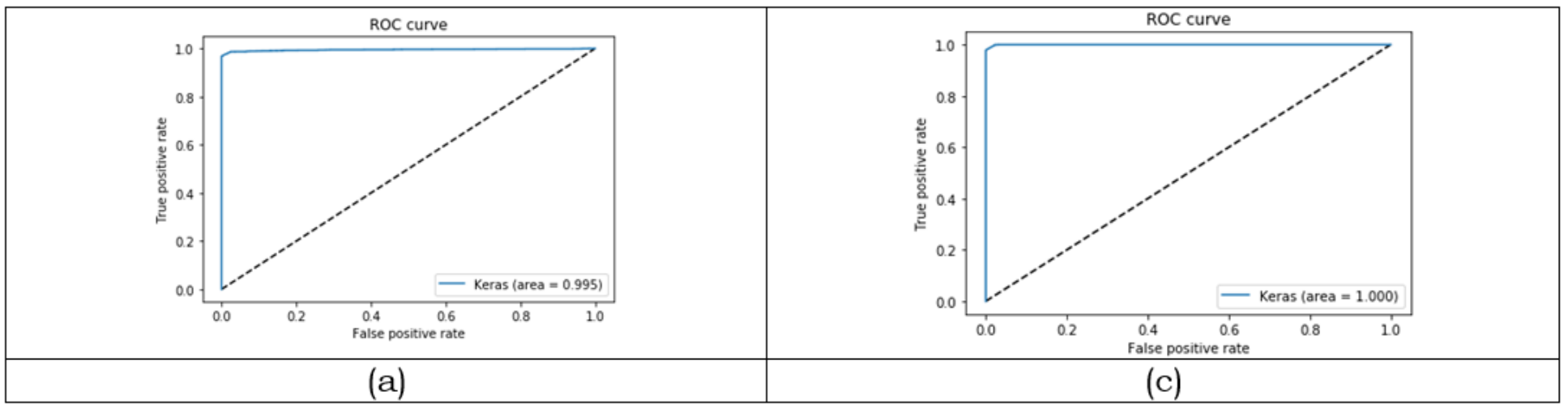

\section{Figure 9}

ROC curves of (a) NSL KDD dataset using GRU (Traditional) (b) NSL KDD dataset using GRU (Proposed) 